\title{
Acoustic scattering from a stiffened finite cylindrical shell with external rings
}

\author{
Fulin Zhou ${ }^{1,2}$, Jun Fan ${ }^{1,2, *}$, and Bin Wang ${ }^{1,2}$ \\ ${ }^{1}$ Collaborative Innovation Center for Advanced Ship and Deep-Sea Exploration, Shanghai Jiao Tong University, 200240 Shanghai, \\ China \\ ${ }^{2}$ State Key Laboratory of Ocean Engineering, Shanghai Jiao Tong University, 200240 Shanghai, China
}

\begin{abstract}
Studying the interaction of sound with cylindrical shells immersed in water is essential and helpful to improving underwater target detection and classification algorithms. Elastic cylindrical shells often occur as part of double-layered shell and have been widely used in marine and aerospace area. Acoustic waves are easy to be transmitted through the outer shell to the interior especially at low frequencies, thus directly being scattered by the inner shell and the rings in water between double-layered shells. Therefore, the externally ring-stiffened cylindrical shell is investigated in this paper. An experiment was conducted that measured the acoustic scattering. A hybrid 2-D/3-D finite-element modelling technique is employed to numerically calculate the scattering characteristics. Good qualitative agreement is found between numerical calculations and experimental measurement. An approximate analytical expression is given explicitly to identify the Bragg wave trajectories in the frequency-angle spectrum. It also has been shown that the rings not only affect the dynamic response of shell and indirectly influence the exterior scattered field, but also become direct acoustic scatterers in water and increase the target cross section especially at oblique incidence.
\end{abstract}

\section{Introduction}

Elastic cylindrical shells often occur as part of double shell constructions and have been widely used in marine and aerospace research. Previous studies focused on the scattering from the internally ring-stiffened cylindrical shells [1-5] and other inner structures [6-9]. Ordinarily, various inclusions can be modelled as beams, rods, or thin plates, which are equivalent to the forces and bending moments coupling with the outer shell, and each type of resonance can be associated with a particular type of interaction between the shell and the inclusions.

Double-layered shells are structurally connected to each other by common frames or rings thereby significantly affecting the sound scattered or radiated from the shells. Based on the thin shell theory, Skelton [10] examined the sound scattering from two concentric cylindrical shells, in which the internal rings indirectly influence the exterior acoustic scattered field through the shells' dynamic response. In target detection, acoustic waves are easily transmitted through the outer shell to the interior, especially at low frequencies, and the waves are then scattered by the inner shell and the rings in the water area between double-layered shells. In this paper, the acoustic scattering from an externally ring-stiffened cylindrical shell is investigated as a simplification of double- layered shells.

Numerical methods are generally used to calculate the structural-acoustic response of complex structures.
An efficient finite element (FE) model with perfectly matched layers (PMLs) was proposed for calculating the radiation and scattering from axisymmetric objects [11]. The 3-D problem is decomposed into 2-D Fourier modal subproblems to solve the target scattering in the near field; next, the scattered pressure in the far field is calculated by 3-D propagation model. The hybrid 2-D/3D FE model has been applied to investigate sound propagation in the ocean and the scattering from complex objects [12].

The scattering from an externally ring-stiffened cylindrical shell is investigated in this study. A tank experiment was conducted in Sec.2. The hybrid 2-D/3-D FE modelling is introduced and applied to calculate the frequency-angle spectrum in Sec.3. Approximate theoretical analysis is performed on the experimental and numerical results in Sec.4. Finally, conclusions are presented in Sec.5.

\section{Free-field tank experiments}

The experiment was conducted in a tank facility located at Shanghai Jiao Tong University. The facility consists of a large quadrate tank with dimensions of $5 \times 5 \times 5-\mathrm{m}^{3}$, filled with water to a depth of approximately $4.5 \mathrm{~m}$. The experimental configuration is shown in Fig.1. An externally ring-stiffened cylindrical steel shell was manufactured, which is closed by two flat endcaps. The shell has an outer radius $\mathrm{a}=3.12 \mathrm{~cm}$, total length $L=42$

\footnotetext{
Corresponding author: fanjun@sjtu.edu.cn
} 
$\mathrm{cm}$, thickness $h_{1}=1 \mathrm{~mm}$, and ratios of $h_{1} / a=0.032$ and $a / L=0.149$. The rings have a height $H=1.3 \mathrm{~cm}$, thickness $h_{2}=0.9 \mathrm{~mm}$, and rib spacing $d=2.1 \mathrm{~cm}$. The elastic parameters are consistent with 304 stainless steel with $E=191 \mathrm{GPa}, \sigma=0.279$ and $\rho=7570 \mathrm{~kg} / \mathrm{m}^{3}$. The external fluid is water, with $\mathrm{c}_{0}=1470 \mathrm{~m} / \mathrm{s}$ and $\rho_{0}=1000 \mathrm{~kg} / \mathrm{m}^{3}$.

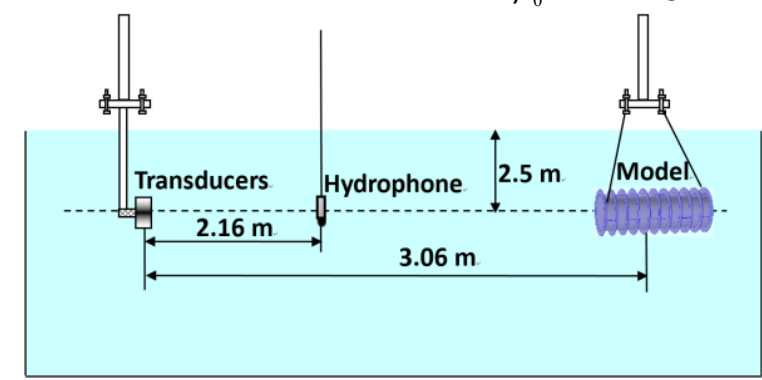

Fig. 1. Experimental configuration.

The dimensionless frequency range is $2.7<k a<32.0$. Considering the broad bandwidth $20-240 \mathrm{kHz}(\mathrm{ka}=2.7$ 32.0), we employed four transducers with bandwidths of $20-40 \mathrm{kHz}, 40-80 \mathrm{kHz}, 60-120 \mathrm{kHz}$, and $120-240 \mathrm{kHz}$. A standard Teledyne RESON hydrophone (TC4014) was used, and its sensitivity is $-186 \mathrm{~dB} \pm 3 \mathrm{~dB}$ re $1 \mathrm{~V} / \mu \mathrm{Pa}$ over the frequency range $15 \mathrm{~Hz}$ to $480 \mathrm{kHz}$. The whole experimental spectra were completed by overlapping and stitching together the four slices of data. The entire measurement system is powered by a high-capacity uninterruptible power supply (UPS) to get rid of the power line interference.

Experimental object was rotated horizontally by drivers at a speed of $60^{\circ} / \mathrm{min}$ in sequence to obtain the full-azimuth scattering characteristics. Continuous data acquisition was achieved in a time interval of $0.5 \mathrm{~s}$ to ensure adequate resolution of the experimental spectra. Having obtained the direct waves and reflections from target through appropriate time windows, we performed an FFT on the time signals for each incidence angle to obtain the experimental frequency-angle spectrum.

Based on the above data processing methods, the experimental frequency-angle spectrum of the externally ring-stiffened cylindrical shell is calculated, as shown in Fig.2(a). Distinct highlights are observed. Considering previous studies on ribbed structures (e.g., Ref. [4,5]), most of these bright arcs are naturally considered to be from Bragg and Bloch-Floquet wave scattering.

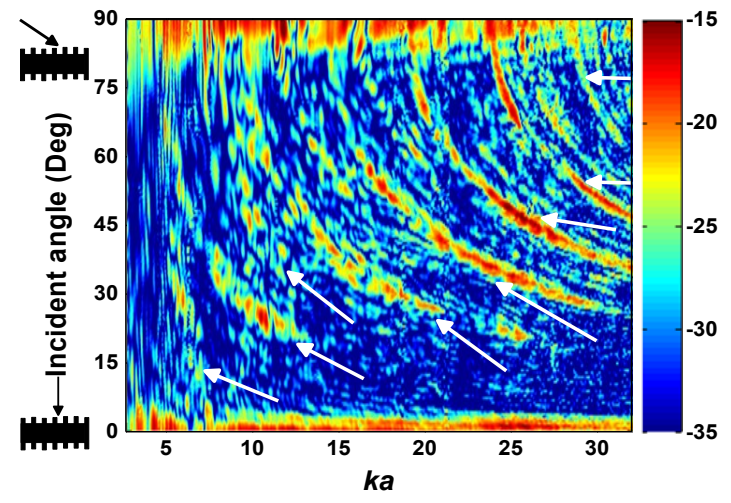

Fig. 2. Experimental frequency-angle spectrum of the externally ring-stiffened cylindrical shell.

\section{Hybrid 2-D/3-D finite element modelling}

Consider a harmonic plane wave incident upon a thin cylindrical shell submerged in water and assume that the incident wave is parallel to the $X O Y$ plane with an incident angle $\theta_{i}$ (see Fig.1). The origin $O$ is located at the centre of the shell. The interior of the shell is in vacuo. A set of rings are seamlessly connected to the outside surface of the shell. Therefore, the target subjects to an asymmetrical forcing field. The harmonic time dependence $e^{-i \omega t}$ will be omitted throughout.

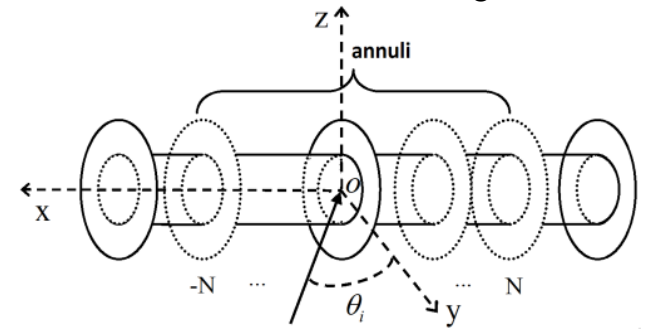

Fig. 3. Schematic diagram of the externally ring-stiffened cylindrical shell model.

The 3-D wave equations in the fluid and linear elastodynamic equations in the solid are broken into Fourier series in cylindrical coordinates using the model superposition technique [11]. The solid displacement $(u, v, w)$ and the pressure $p$ are decomposed in the same manner:

$$
\left(\begin{array}{c}
u(r, \varphi, z) \\
v(r, \varphi, z) \\
w(r, \varphi, z) \\
p(r, \varphi, z)
\end{array}\right)=\left(\begin{array}{l}
u_{n}(r, z) e^{i n \varphi} \\
v_{n}(r, z) e^{i n \varphi} \\
w_{n}(r, z) e^{i n \theta} \\
p_{n}(r, z) e^{i n \varphi}
\end{array}\right)
$$

where $n$ represents the circumferential order and $\varphi$ is the circumferential angle $(0 \leq \varphi<2 \pi)$.

In the numerical calculations, the series expansion is truncated at a modal order $n=N_{j}$, where $N_{j}$ is the nearest integer to $1.6 k r_{\max }\left|\cos \theta_{i}\right|$ and $r_{\max }$ corresponds to the largest radial coordinate of the model [11].

To simulate the free field, a Berenger PML with cylindrical coordinates is implemented here in the azimuthal modal decomposition FE scheme [13]. The far-field pressure $p_{s}$ can be calculated in accordance with the discrete form of the Helmholtz integral [12]. Finally, the target strength (TS) takes the following form:

$$
T S=20 \log \left(\frac{r\left|p_{s}\right|}{r_{0}\left|p_{\text {inc }}\right|}\right)
$$

where $r$ is the distance between the target center and the far field receiver location $(\mathrm{m}), r_{0}$ is a reference distance $(1 \mathrm{~m})$, and the quantity $\left|p_{\text {inc }}\right|$ is the amplitude of the incident plane wave at the center of the target. The TS in the $X O Y$ plane is a function of the frequency and incident angle.

The frequency-angle spectrum of the TS for the externally ring-stiffened cylindrical shell is calculated in which the parameters are the same as experimental 
model, as shown in Fig.4. Good agreement can be found between the experimental and numerical results.

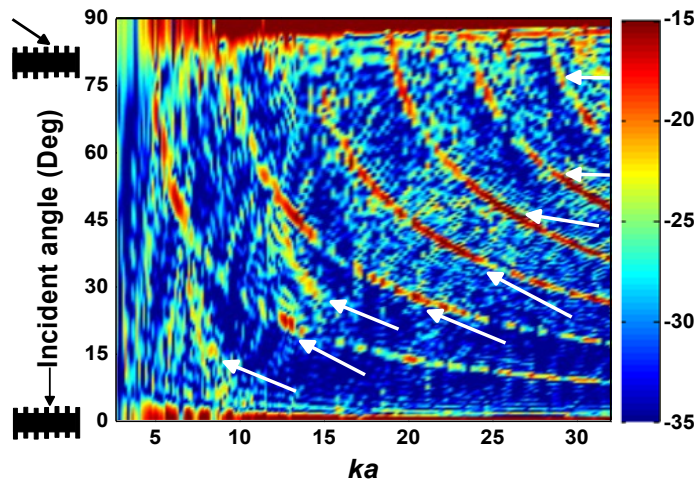

Fig. 4. Simulated frequency-angle spectrum of the externally ring-stiffened cylindrical shell by FEM.

\section{Approximate theoretical analysis}

Similar to a periodically ribbed plate, the Bragg scattering phenomenon of an infinite ring-stiffened cylindrical shell can be described in Fig.5.

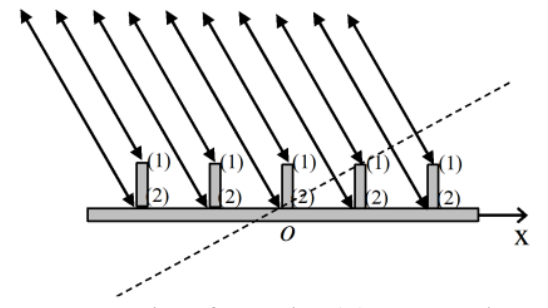

Fig. 5. Bragg scattering from the (1) outer edges and (2) inner edges of the stiffeners.

Bragg scattering arises from the geometrical reflections of the incident wave on the inner and outer edges of the rings. These two parts have different arrival times, whereas their trajectories in frequency-angle spectrum are consistent and can be evaluated as follows:

$$
2 k_{0} d \sin \theta_{i}=2 m \pi
$$

where $k_{0}$ is the wavenumber of the incident plane wave, and $d$ is the ring spacing. Theoretical Bragg trajectories of the first six orders are calculated as shown in Fig.6.

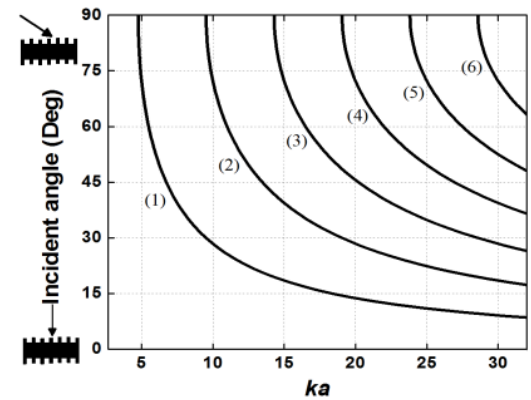

Fig. 6. Theoretical Bragg trajectories of the first six orders (1)-(6).

Based on theoretical Bragg and BFW trajectories by the approximate theoretical method, we can identify some of the bright arcs in experimental and numerical frequency-angle spectrua in Fig. 2 and Fig.4. Taking the experimental spectrum as an example, the identification result is shown in Fig.10. Note that not all of the theoretical prediction curves can be identified in the spectrum. The reason may be that external rings are modelled as solid elements and the complex echoes due to the elastic effects of rings cover the theoretical trajectories partly. External rings transmit the coupling forces and moments to the double-layered shells and contribute to their dynamic response, which indirectly influence the exterior acoustic field. Moreover, these rings become the direct elastic scatterers in water and lead to multiple scatterings.

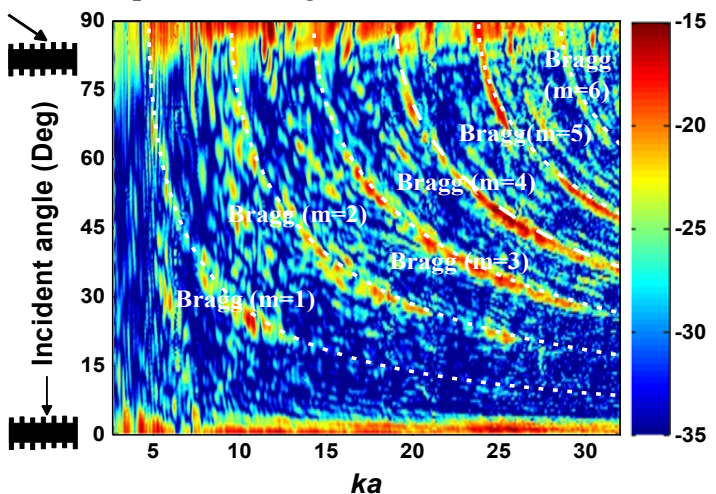

Fig. 10. Identification of Bragg and BFW wave trajectories in frequency-angle spectrum.

\section{Conclusions}

The backscattering from an externally ring-stiffened cylindrical shell was measured in a free-field tank experiment, revealing the complexity of the acoustic response from the target. A hybrid 2-D/3-D finite element modelling technique was employed to efficiently calculate the frequency-angle spectrum. Good agreement can be found between the experimental and numerical results. The Bragg wave trajectories in the frequency-angle spectrum were identified using approximate theoretical analysis. Compared with the case of a ribbed plate, the BFWs in a ring-stiffened cylindrical shell include three kinds of elastic waves: flexural wave $\mathrm{a}_{0}$, compressional wave $\mathrm{s}_{0}$ and shear wave $\mathrm{T}_{0}$. However, not all of the theoretical prediction curves can be identified in the spectrum. In addition to the indirect influence of the forces and bending moments on the shell similar to internal rings, the external rings in water act as the direct acoustic objects that increase the target cross section, especially at an oblique incidence.

The work is supported by the National Science Foundation for a Distinguished Young Scholars of China Grant (No. 11504233). The authors are grateful to Professor Weilin Tang, Dr Yunzhe Tong, and Dr. Bo Liu for helpful discussions. We also appreciate the help of Kai Xu and Dr. Kaiqi Zhao during the experiment.

\section{References}

1. P. J. Moser, H. Überall, J. R. Yuan, Acoust. Soc. Am., 94 (1993)

2. D. M. Photiadis, J. A. Bucaro, B. H. Houston, Acoust. Soc. Am., 96 (1994) 
3. B. H. Houston, J. A. Bucaro, D. M. Photiadis, Acoust. Soc. Am., 98 (1995)

4. M. Tran-Van-Nhieu, Acoust. Soc. Am., 110 (2001)

5. R. Liétard, D. Decultot, G. Maze, M. Tran-VanNhieu, Acoust. Soc. Am., 118 (2005)

6. Y. P. Guo, Acoust. Soc. Am., 95 (1994)

7. J. M. Cuschieri, D. Feit, Acoust. Soc. Am., 98 (1995)

8. J. M. Cuschieri, D. Feit, Acoust. Soc. Am., 98 (1995)

9. A. Pan, J. Fan and B. Wang, Acoust. Soc. Am., 134 (2013)

10. E. A. Skelton, J. Sound Vib., 181 (1995)

11. M. Zampolli, A. Tesei, F. B. Jensen, N. Malm, J. B. Blottman III, Acoust. Soc. Am., 122 (2007)

12. A. L. España, K. L. Williams, D. S. Plotnick and P. L. Marston, Acoust. Soc. Am., 136 (2014)

13. J. P. Berenger, J. Comput. Phys., 114 (1994) 